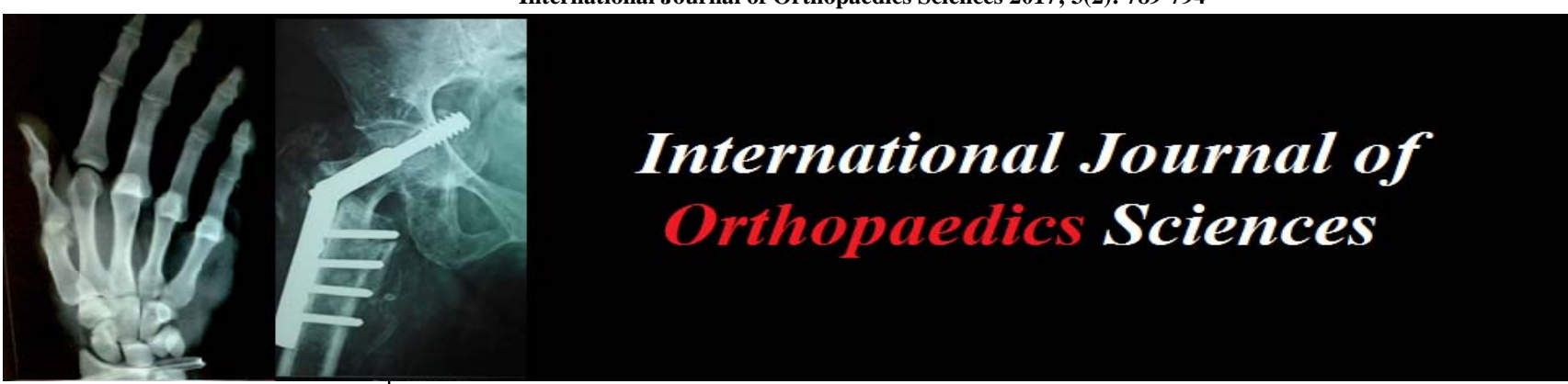

ISSN: $2395-1958$

IJOS 2017; 3(2): 789-794

(C) 2017 IJOS

www.orthopaper.com

Received: 24-02-2017

Accepted: 25-03-2017

Dr. Prerak Yadav

Senior Resident. Department of

Orthopaedics. Government

Medical College, Bhavnagar,

Gujarat, India

Dr. Vatsal Parmar

Junior Resident. Department of

Orthopaedics. Government

Medical College, Bhavnagar,

Gujarat, India
Correspondence

Dr Prerak Yadav

Senior Resident. Department of

Orthopaedics. Government

Medical College, Bhavnagar,

Gujarat, India

\section{Outcomes of local injections of platelet rich plasma in various chronic tendinopathies}

\author{
Dr. Prerak Yadav and Dr. Vatsal Parmar \\ DOI: $\underline{\text { http://dx.doi.org/10.22271/ortho.2017.v3.i2i.84 }}$
}

Abstract

Introduction: Tendinopathy is a repetitive strain injury caused by repetitive overuse of the muscles of the body. It causes pain and functional impairment in daily activities. The treatment of this condition includes conservative therapy and surgical interventions. The effectiveness of oral nonsteroidal antiinflammatory agents, topical and injectable medications including corticosteroids and botulinum toxins, splinting, physical therapy, and iontophoresis has been evaluated in many studies. Platelet-rich plasma (PRP) therapy has been utilized for nearly two decades to treat degenerative joint disease and speed recovery from soft tissue injuries to tendons, muscles and ligaments.

Aim: The present study aims at evaluating the results of PRP treatment in chronic tendinopathy and assessment of joint function and functional abilities of patients in follow up studies.

Materials and Methods: This study was a prospective, comparative, single blind study, carried out in 30 patient. The patients were assessed pre procedure and post procedure in form of range of movements, pain, tenderness, joint stiffness and early mobilization. Quantity of PRP given was $2 \mathrm{ml}$ in $<6$ month chronicity and $3 \mathrm{ml}$ in $>6$ month chronicity. The functional outcome of PRP treatment was evaluated with visual analogue score.

Results and Discussion: The functional outcome i.e. pain improvement in our study was evaluated using VAS (Visual analogue Score). About $64 \%$ patients in our study attained excellent VAS scores. Our results were comparable to the results obtained by Mishra and his colleagues ${ }^{8}$, in 2006, where patients who had received PRP treatment showed 60\% improvement in their VAAS pain score.

Conclusion: Our study had shown good to excellent symptom improvement in $90 \%$ of patient. Almost all patient treated with PRP had good range of motion and pain relief at the end of the study and most of patients were able to do their routine or previous work and activities without any difficulty.

Being newer techniques, PRP treatment requires further evaluation and there is a steep learning curve

Keywords: Local injections, platelet rich plasma, various chronic tendinopathies, joint stiffness

\section{Introduction}

Platelet-rich plasma (PRP) therapy has been utilized for nearly two decades to treat degenerative joint disease and speed recovery from soft tissue injuries to tendons, muscles and ligaments. By isolating growth-factor rich platelets from the patient's blood and injecting this potent plasma into site of injury, this therapy has shown promise in its ability to catalyze the growth of new tissue and bone cells and thus promote healing.

Tendinopathy is a repetitive strain injury caused by repetitive overuse of the muscles of the body. It causes pain and functional impairment in daily activities. The treatment of this condition includes conservative therapy and surgical interventions. The effectiveness of oral nonsteroidal anti-inflammatory agents, topical and injectable medications including corticosteroids and botulinum toxins, splinting, physical therapy, and iontophoresis has been evaluated in many studies. However, these traditional therapies do not alter the tendon's inherent poor healing properties secondary to poor vascularization ${ }^{[1]}$. Short coming of conservatively treated chronic tendinopathy include Joint stiffness near the affected joint, Pain, tenderness, redness, warmth at affected tendon, Crepitus or crunchy sound or feeling when the tendon is used, Pain and stiffness that may be worse during the night or when getting up in the morning Given the inherent nature of the tendon, new treatment options including platelets rich plasma (PRP), autologous blood, and prolotherapy ${ }^{[2]}$ are aimed at inducing inflammation rather than suppressing it. 
PRP is quite a new treatment used for chronic tendinopathy. Platelet rich plasma is defined a volume of the plasma fraction of autologous blood having a platelet concentration above baseline [3] PRP contain platelets, and these platelets have strong growth factors and granules that have critical role in the healing process of chronic injuries. Due to higher concentration of platelets in PRP than whole blood, it was shown to have greater effect in the repair process in treatment of chronic nonhealing tendinopathy. Benefits of PRP treatment include better healing of tendon, early mobilization, prevention of stiffness of joint, good range motion of joint.

\section{Aims and Objectives}

The present study aims at evaluating the results of PRP treatment in chronic tendinopathy and assessment of joint function and functional abilities of patients in follow up studies.

\section{Materials and Methods}

This study was a prospective, comparative, single blind study, carried out at Sir Takhtsinhji General Hospital, Bhavnagar carried out from January 2016 to June 2016 with a follow up of 6 months.

After institutional review board approval and informed written consent from patients, this prospective case controlled clinical study was carried out in 30 patients. All patient needed to undergo anterior-posterior (AP) and lateral radiographs as well as ultrasound and MRI to identify swelling, tears, calcification in tendon, bursae, muscle and bone.

The patients were assessed pre procedure and post procedure in form of range of movements, pain, tenderness, joint stiffness and early mobilization.

After thorough pre procedure evaluation and required investigations, patients meeting the following inclusion criteria were included in the study.

1. Patient willing to provide written informed consent to participate in the study

2. Patients with chronic clinically diagnosed tendinopathy (based on symptoms, site of tenderness, and pain elicited with resisted active movement)

3. Patients having duration of symptoms more than 3 months and pain severity.

Patients falling in the below mentioned exclusion criteria were excluded from the study:

1. Presence of other systemic disorder URTI, high blood pressure, uncontrolled diabetes, poor respiration.

2. Patients not willing to provide written informed consent to participate in the study.

3. Patients having history of trauma, any platelet dysfunction syndrome (Critical thrombocytopenia), any other coagulopathies (such as hypofibrinogenemia), local infection at the site of the procedure, any recent febrile or infectious disease,

Patients were assessed preprocedure for severity of signs and symptoms, which were compared with post procedure signs and symptoms. Patients were also evaluated for following variables:

- Range of joint movement

- Severity of pain

- Stability of joint

- Ability to perform special function
Quantity of PRP given was $2 \mathrm{ml}$ in $<6$ month chronicity and $3 \mathrm{ml}$ in $>6$ month chronicity.

\section{PRP Preparation}

The patient was placed in an appropriate and comfortable position that allows for sterility and access to the site of injection.

At first, $20 \mathrm{cc}$ of venous blood was drawn with aseptic technique from venous antecubital vein and transferred to the centrifuge.

For preparing $2 \mathrm{~mL}$ of PRP with concentration of 4-6 times the average normal values, $20 \mathrm{~mL}$ of blood was first collected from the patient's upper limb cubital vein using an $18 \mathrm{G}$ needle. Then $2 \mathrm{ml}$ of ACD-A was added to the sample as an anticoagulant. $1 \mathrm{~mL}$ of the blood sample was sent for complete blood count. The rest of the sample passed two stages of centrifuge (first with $1600 \mathrm{rpm}$ for 15 minutes for separation of erythrocytes and next with $2800 \mathrm{rpm}$ for $7 \mathrm{~min}-$ utes in order to concentrate platelets). The final product was 2 $\mathrm{ml}$ of PRP containing leukocytes. The PRP quantification and qualification procedure was performed using laboratory analyzer Sysmex KX 21 and if approved, the injection was proceeded ${ }^{[4]}$.

\section{PRP Injection}

The skin of the injection site was prepped and draped and the liquid PRP was injected in a sterile condition using a $22 \mathrm{G}$ needle at maximal tender point at elbow using a peppering technique spreading in a clock- like manner to achieve a more expansive zone of delivery ${ }^{[4]}$.

\section{Complications \\ 1. Infection \\ 2. Vascular injury \\ 3. Nerve injury \\ 4. Allergic reaction \\ 5. Skin discoloration}

\section{Outcome}

1. Evaluate the functional outcome of prp treatment in various chronic with visual analogue score.

2. Percentage of radiography abnormality by X-Ray by MPTA and PPTA.

3. Percentage of rate and type of complication after the treatment under study.

\section{Statistical analysis}

The study contained total sample size of 30 patients. Each variable was recorded and analyzed using Jindal Sigma Statistical Software ver.2.0. Detailed analysis was carried out with the required mean and standard deviation of the respected variables. The association between variables was analyzed with independent student's $\mathrm{T}$ test and paired $\mathrm{T}$ test for quantitative variables and by chi square test for qualitative variables.

The significant association was considered only when $p$ value is $<0.05$.

\section{Evaluation of Outcome}

For evaluating the functional outcome of PRP treatment we used the visual analouge score ${ }^{[5,6]}$. This system takes into account the following parameters: 
Vas score

\begin{tabular}{|c|c|c|}
\hline Rating & Score & Result \\
\hline No pain & 0 & Excellent \\
\hline Mild pain & $1-3$ & Good \\
\hline Moderate pain & $4-6$ & Fair \\
\hline Severe pain & $7-10$ & Poor \\
\hline
\end{tabular}

\section{Results}

\section{Age Distribution}

The age group varied form 30 years to 70 years with the mean age of 40 years. Incidence of fracture was observed maximum between 30 - 50 years of age.

Table 1

\begin{tabular}{|c|c|c|}
\hline Age Group & Number of Cases & Percentage \\
\hline $30-40$ Years & 9 & $30 \%$ \\
\hline $40-50$ & 10 & $33 \%$ \\
\hline $50-60$ & 1 & $4 \%$ \\
\hline $60-70$ & 10 & $33 \%$ \\
\hline
\end{tabular}

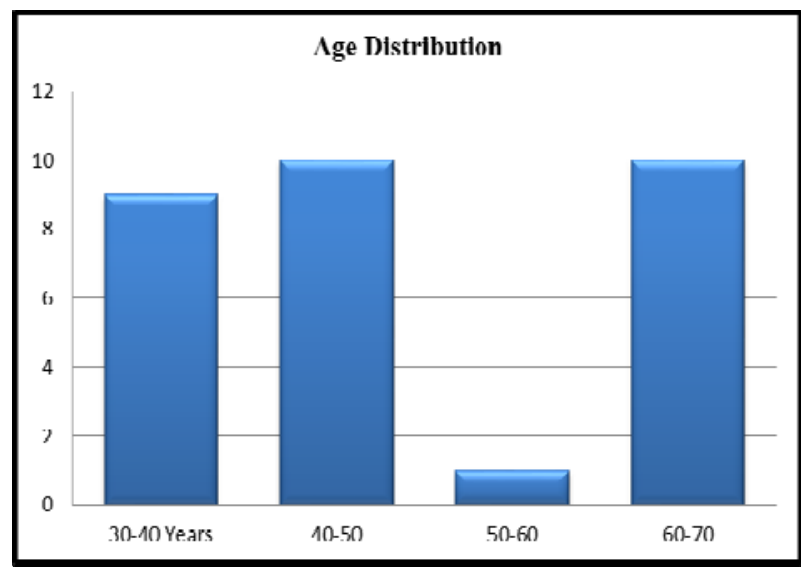

Graph 01

\section{Sex Distribution}

Among the 30 cases, females were slightly predominant

Table 2

\begin{tabular}{|c|c|c|}
\hline Sex & Number of Cases & Percentage \\
\hline Male & 14 & $47 \%$ \\
\hline Female & 16 & $53 \%$ \\
\hline
\end{tabular}

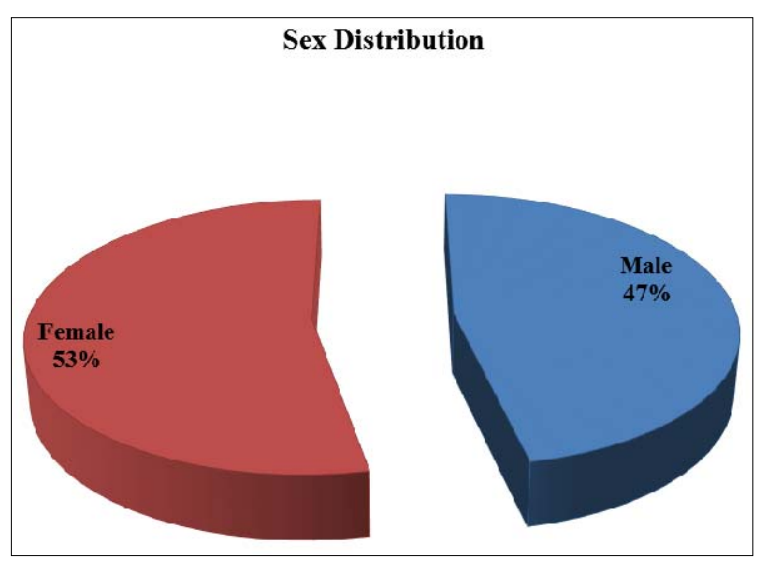

Graph 02

\section{Side of Tendinopathy}

Left side was more common in our series.
Table 3

\begin{tabular}{|c|c|c|}
\hline Side & Number of Cases & Percentage \\
\hline Right & 14 & $47 \%$ \\
\hline Left & 16 & $53 \%$ \\
\hline
\end{tabular}

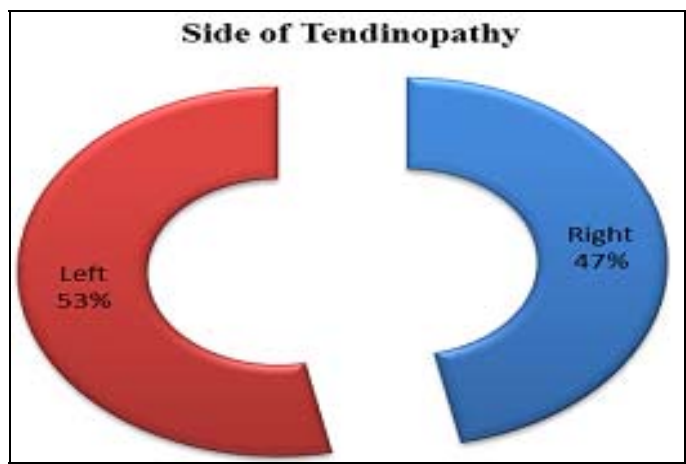

Graph 03

\section{Type of Tendinopathy}

Frozen Shoulder was more common in our series.

Table 4

\begin{tabular}{|c|c|c|}
\hline Type of Tendinopathy & Number of Cases & Percentage \% \\
\hline Frozen Shoulder & 13 & $44 \%$ \\
\hline Tennis Elbow & 7 & $23 \%$ \\
\hline Planter Fascitis & 10 & $33 \%$ \\
\hline
\end{tabular}

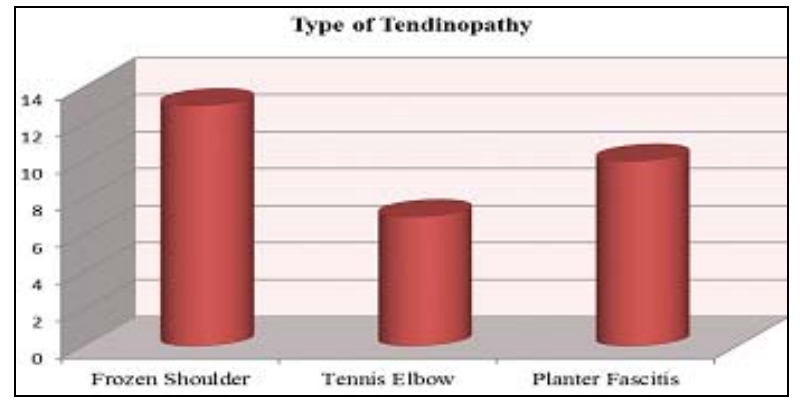

Graph 04

\section{Chronicity of Tendinopathy}

Table 5

\begin{tabular}{|c|c|c|}
\hline Chronicity & Number of Cases & Percentage \% \\
\hline$<6$ Month & 12 & $40 \%$ \\
\hline$>6$ Month & 13 & $60 \%$ \\
\hline
\end{tabular}

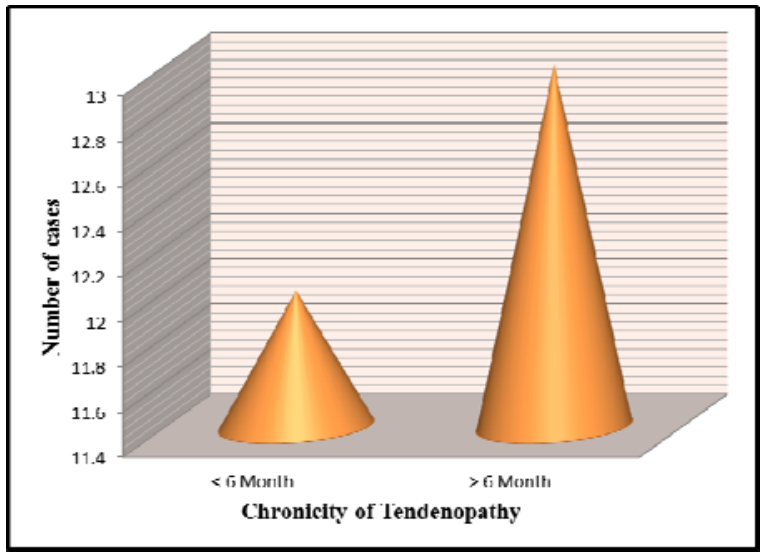

Graph 05 


\section{Associated Injuries}

Table 6

\begin{tabular}{|c|c|c|}
\hline Type & Number of Cases & Percentage \% \\
\hline F racture at local site & 02 & $6 \%$ \\
\hline History of RTA & 01 & $4 \%$ \\
\hline History of FD & 03 & $10 \%$ \\
\hline No Past history of any injury & 24 & $80 \%$ \\
\hline
\end{tabular}

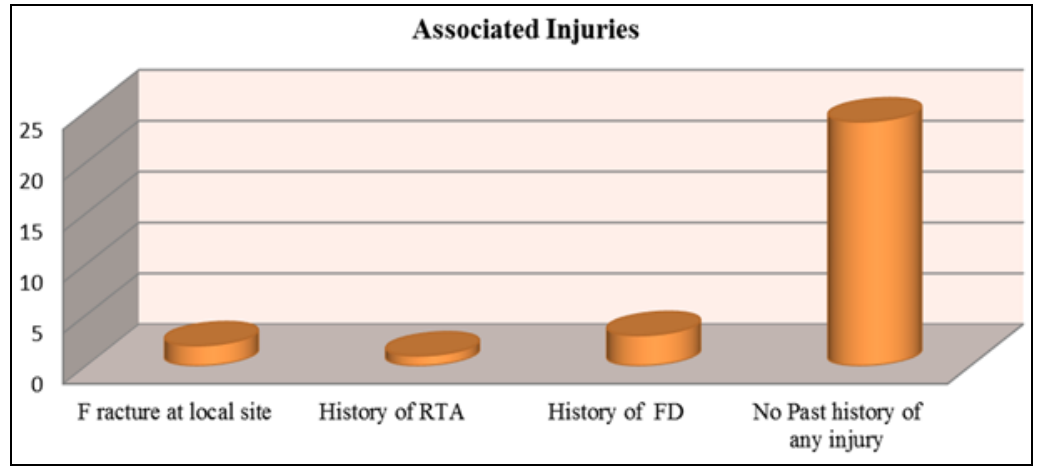

Graph 06

\section{Time Interval for Improvement}

Table 7

\begin{tabular}{|c|c|c|c|c|c|}
\hline \multirow{2}{*}{ Days } & \multicolumn{2}{|c|}{ Number of Cases } & \multirow{2}{*}{ Total } & \multirow{2}{*}{ Percentage \% } \\
\cline { 2 - 4 } & FS & TE & PF & & \\
\hline 5 to 10 days & 05 & 03 & 03 & 11 & $37 \%$ \\
\hline 10 to 15 days & 05 & 03 & 04 & 12 & $40 \%$ \\
\hline 15 to 30 days & 03 & 01 & 03 & 07 & $23 \%$ \\
\hline
\end{tabular}

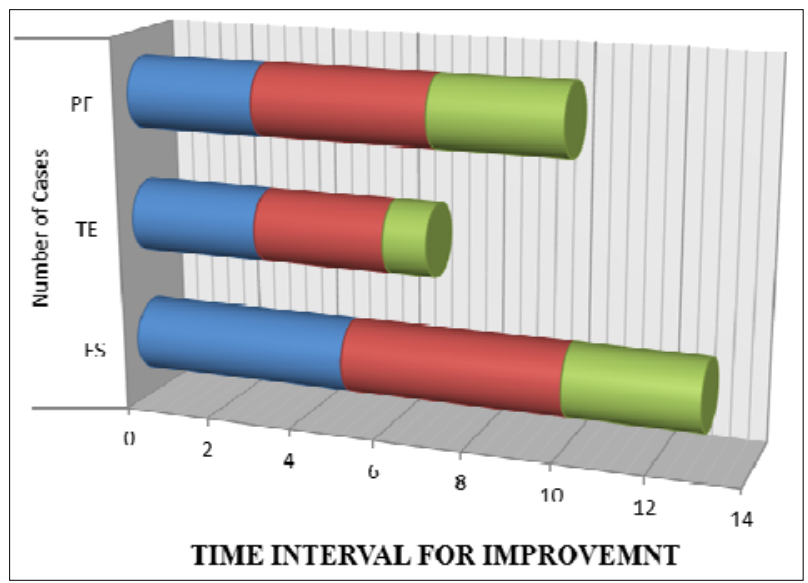

Graph 07

\section{Complications}

Table 8

\begin{tabular}{|c|c|c|c|}
\hline \multirow{2}{*}{ Complications } & \multicolumn{3}{|c|}{ Number of Cases } \\
\cline { 2 - 4 } & FS & TE & PF \\
\hline Infections & 0 & 0 & 0 \\
\hline Vascular Injury & 0 & 0 & 0 \\
\hline Nerve Injury & 0 & 0 & 0 \\
\hline Allergic Reaction & 01 & 0 & 0 \\
\hline Skin Discoloration & 02 & 0 & 0 \\
\hline
\end{tabular}

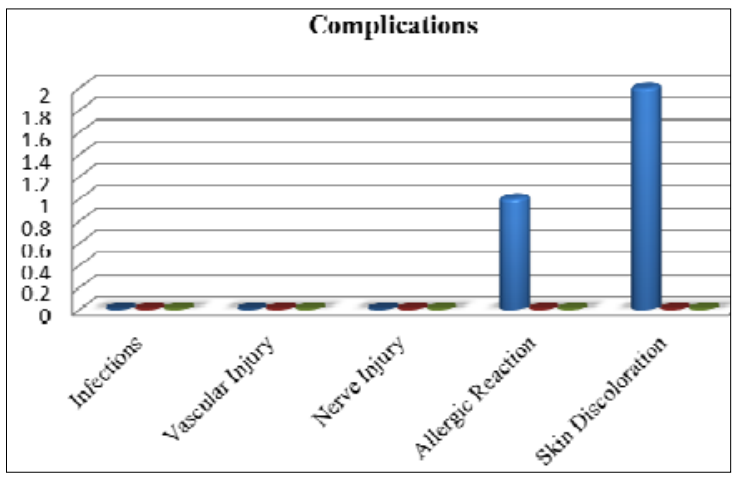

Graph 08

\section{Analysis of functional outcome}

\section{Overall Results}

The Analysis was done using visual Analouge Score and the following results were obtained.

Table 9

\begin{tabular}{|l|l|l|}
\hline Grading & Number of Cases & Percentage \% \\
\hline Excellent & 19 & $64 \%$ \\
\hline Good & 08 & $26 \%$ \\
\hline Fair & 02 & $6 \%$ \\
\hline Poor & 01 & $4 \%$ \\
\hline
\end{tabular}

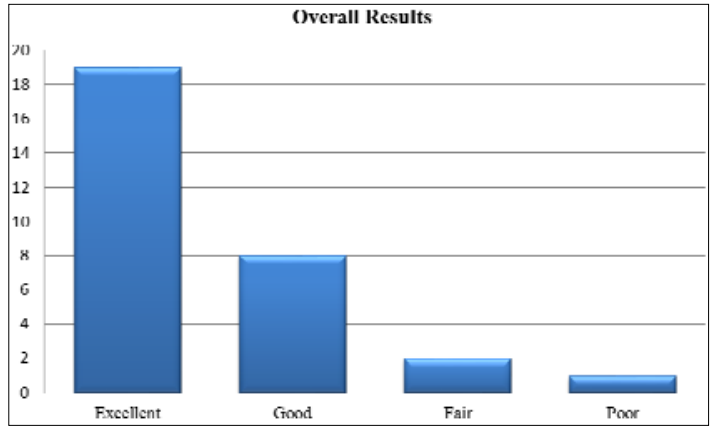

Graph 09 


\section{Results According to Tendinopathy}

Table 10

\begin{tabular}{|c|c|c|c|c|c|c|}
\hline \multirow{2}{*}{ Grading } & \multicolumn{7}{|c|}{ Number of Cases } \\
\cline { 2 - 7 } & FS & $\mathbf{\%}$ & TE & $\mathbf{\%}$ & PF & \% \\
\hline Excellent & 09 & $30 \%$ & 05 & $16 \%$ & 05 & $16 \%$ \\
\hline Good & 04 & $14 \%$ & 02 & $06 \%$ & 02 & $06 \%$ \\
\hline Fair & 02 & $06 \%$ & 00 & $00 \%$ & 00 & $00 \%$ \\
\hline Poor & 00 & $00 \%$ & 00 & $00 \%$ & 01 & $04 \%$ \\
\hline
\end{tabular}

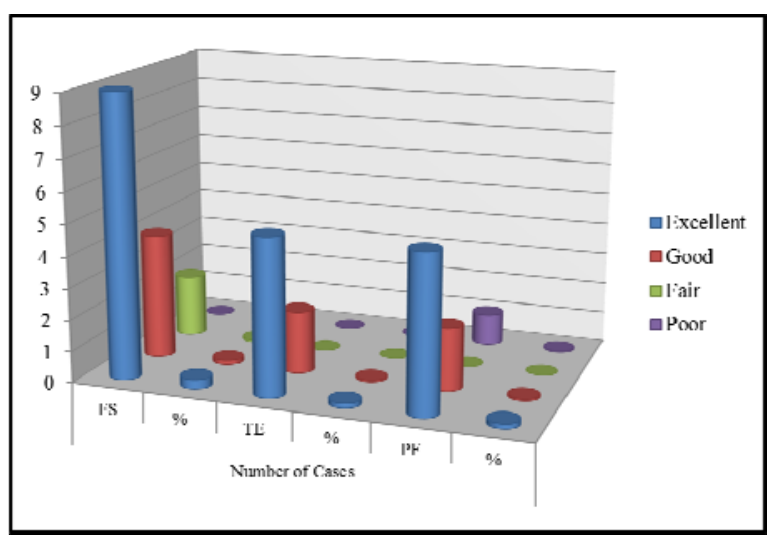

Graph 10

\section{Discussion}

The aim of our study was to evaluate the results PRP treatment in chronic tendinopathy and assessment of joint function and functional abilities of patient in follow up studies Chronic conditions such as Achilles tendinopathy (tendinosis) are not uncommon, but are extremely difficult sports injuries to treat. The pathophysiology of tendinopathy-the term used to refer to chronic inflammation of the tendon (as distinguished from tendinitis, which refers to the acute inflammatory state)-continues to elude physicians studying the issue. Therapeutic treatment of the disease is difficult, especially in the chronic conditions.

The most common treatment approach still remains the conservative approach, although the percentage of positive results in chronic conditions is about $50 \%{ }^{[7]}$. Oral and topical anti-inflammatory medications, copper bracelets, high- or low-intensity pulsed ultrasound, extracorporeal shock wave therapy (ESWT), and injected platelet-rich plasma (PRP) have all been used to speed recovery. Basic science studies seem to support the application of PRP for the treatment of problems related to tendons. Several limitations exist in these models, and healthy tendon cultures exposed to an agent may respond differently than an intact chronically diseased tendon. Thus, drawing clinical conclusions from these studies is difficult.

Unfortunately, a chronic tendon injury model is difficult to reproduce and is not likely to have identical pathophysiology compared to tendinosis. PRP has been studied clinically mostly in the elbow and Achilles tendon, and some studies seem to show it has some benefit. Based upon clinical study alone, we cannot yet categorically conclude that PRP is beneficial for all conditions related to tendinopathy.

Also, combined therapy (such as using ESWT with PRP) may actually be more effective than a single modality therapy. PRP makes sense intuitively and, other than cost, has very little downside.

In addition PRP may have some anti-nociceptive effect that is still poorly defined. Many of our patients have rapid resolution of symptoms that cannot possibly be explained by resolution of the pathologic findings associated with a diseased tendon an opportunity theoretically exists for improvement by injecting PRP to stimulate angiogenic infiltration and remodeling by tenocytes. PRP therapy may also facilitate healing tenis elbow tendinopathy.

In our study, we tried to evaluate the functional outcomes of PRP treatment in patients with chronic tendinopathy. The most common type of tendinopathy observed in our series of patients was frozen shoulder $(44 \%)$, followed by plantar fasciitis (33\%), followed by tennis elbow (23\%).

The age group varied form 30 years to 70 years with the mean age of 40 years. This was comparable with the study conducted by Reisaddat et al ${ }^{[4]}$, where the mean age of patients was $46 \pm 7.5$ years. Female predominance was observed in our patient population, which was again comparable to the study conducted by Reisaddat et al. The chronicity observed in both the studies was more than 6 years (14.5 years in the study undertaken by Reisaddat et al). The chronicity can be attributed to the avascularised nature of tendon tissue, which can interfere with the healing procedure and hence result in chronic tendinopathies.

The functional outcome i.e. pain improvement in our study was evaluated using VAS (Visual analogue Score). About $64 \%$ patients in our study attained excellent VAS scores. Our results were comparable to the results obtained by Mishra and his colleagues ${ }^{[8]}$, in 2006, where patients who had received PRP treatment showed $60 \%$ improvement in their VAAS pain score. In both the studies, pain improvement was not measured objectively though.

In a study undertaken by Deans et al ${ }^{[9]}$., they obtained statistically remarkable results in terms of pain, various other symptoms, everyday activities, sports activities, and quality of life in a group of 26 patients with chronic Achilles tendinopathy. In an another study by Ferrero et al ${ }^{[10]}$, a significant and long-term improvement in clinical symptomatology, accompanied by recovery of tendon matrix was observed in a group of 48 patients affected by chronic Achilles and patellar tendinopathies. Charousset et al ${ }^{[11]}$. examined 28 athletes with chronic patellar tendinopathy refractory to nonoperative management, where application of 3 consecutive US- guided PRP injections significantly improved symptoms and function in athletes with chronic patellar tendinopathy and allowed fast recovery to their presymptom sporting level Sanchez et al ${ }^{[12]}$. reported superior results in a group of patients with Achilles Tendon treated with PRP injections and also included a control group to evaluate the results.

Our indications for PRP treatment for tenis elbow in adults are severe symptoms present for more than 3 months that are unresponsive to physical therapy and clinical findings collaborated by changes on magnetic resonance images or ultrasound. The patient must stop using nonsteroidal antiinflammatory drugs (NSAIDs) for at least 1 week prior to the injection and avoid NSAID use for 3 to 4 weeks post injection. The post injection protocol includes standard rehabilitation for strength and functional progress and gradual return to activities over 6 to 8 weeks. Frequent and liberal use of ice, particularly in the early stages, has been helpful in controlling any discomfort from the injection. Criteria for return to normal work include full painless range of motion, ability to tolerate going up and down stairs, and no discomfort as sports progression proceeds.

First of all, it is important to determine what, precisely; you are 'asking' PRP preparations to do in the treatment of tendinopathy. That is, what aspect(s) of the wound healing process are you trying to stimulate? In chronic tendinopathy, 
the tissue repair process has been 'stalled' and treatments have been based on inciting an inflammatory response to allow the body's natural repair process to start over.

Although the increase in growth factor concentration associated with PRP has been used to justify its application in the treatment of tendinopathy, platelets also have the ability to store and rapidly release a variety of other bioactive molecules, including proteases and anti- proteases, adhesion proteins, and inflammatory cytokines. Thus, the PRP injection may provide the inflammatory cytokines needed to incite an acute inflammatory response and kick-start the healing process in a compromised tissue. In addition, the proteins (albumin and globulins) and clotting factors (fibrinogen) present in the plasma portion of PRP are critical components in the early stages of wound healing, such as in creating a provisional fibrin scaffold and stimulating the inflammatory response. Although PRP contains all the elements needed to initiate and support wound repair, the precise indication (timing, dosage, and proposed mechanism of action) has yet to be precisely unraveled.

Finally, even though the natural history of tendinopathy has been extensively studied and prescribed treatments have been guided by clinical signs, the level of tissue pathology and the ability of the tissue to respond to PRP (or for that matter any therapeutic intervention) could vary greatly, even in patients with similar clinical signs. A chronic condition such as tendinopathy may display a varying subset of cellular pathologies that subtly change over time, which may explain the varied results that have been reported.

We have had success using PRP injections for tendinosis patients. The discomfort to patients and the cost are issues, but athletes are often frustrated with the chronic discomfort associated with tendinosis.

PRP injections should be used in conjunction with other treatments, such as rest, ice, anti- inflammatory medication, and physical therapy in a multimodal plan to promote symptom relief and tendon healing.

The effectiveness of PRP in treating tendinosis has not been scientifically proven in human trials. Growth factors associated with certain cells can help promote healing; what sort of cell differentiation PRP promotes is unclear. Our experience is anecdotal. More randomized, double-blind studies are needed with human trials.

\section{Conclusion}

Being newer techniques, PRP treatment require further evaluation and there is a steep learning curve. Even though, conventional treatment is still the most common used and PRP treatment in chronic tendinopathy is an acceptable method and it is relatively newer technique which offers and more efficient alternative in all type of chronic tendinopathy in adults. In our study the average time required for functional recovery was around 15 days. The duration of hospital stay post procedure was less than 1 hour. Our study had shown good to excellent symptom improvement in $90 \%$ of patient. Almost all patient treated with PRP had good range of motion and pain relief at the end of the study and most of patients were able to do their routine or previous work and activities without any difficulty.

\section{References}

1. Andres BM, Murrell GAC. Treatment of Tendinopathy: What Works, What Does Not, and What is on the Horizon. Clinical Orthopaedics and Related Research. 2008;466(7):1539-1554.doi:10.1007/s11999-008-0260-1.
2. Childress MA, Beutler A. Management of chronic tendon injuries. American family physician, 2013; 87(7).

3. Civinini R, Macera A, Nistri L, Redl B, Innocenti M. The use of autologous blood-derived growth factors in bone regeneration. Clinical Cases in Mineral and Bone Metabolism, 2011; 8(1):25-31.

4. Seyed Ahmad Raeissadat, Leyla Sedighipour, Seyed Mansoor Rayegani, Mohammad Hasan Bahrami, Masume Bayat, Rosa Rahimi. Effect of Platelet-Rich Plasma (PRP) versus Autologous Whole Blood on Pain and Function Improvement in Tennis Elbow: A Randomized Clinical Trial, Pain Research and Treatment, 2014, 8. Article ID 191525, doi:10.1155/2014/191525

5. Boonstra AM, Preuper HRS, Reneman MF, Posthumus JB, Stewart RE. Reliability and validity of the visual analogue scale for disability in patients with chronic musculoskeletal pain. International Journal of Rehabilitation Research. 2008; 31(2):165-169.

6. Price DD, Bush FM, Long S, Harkins SW. A comparison of pain measurement characteristics of mechanical visual analogue and simple numerical rating scales. Pain, 1994; 56(2):217-226.

7. Antonio Crescibene, Marcello Napolitano, Raffaella Sbano, Enrico Costabile, Hesham Almolla. Infiltration of Autologous Growth Factors in Chronic Tendinopathies. Journal of Blood Transfusion. 2015, 6 . Article ID 924380, doi:10.1155/2015/924380

8. Mishra A, Pavelko T. Treatment of chronic elbow tendinosis with buffered platelet-rich plasma. The American journal of sports medicine. 2006; 34(11):17741778.

9. Deans VM, Miller A, Ramos J. A prospective series of patients with chronic Achilles tendinopathy treated with autologous-conditioned plasma injections combined with exercise and therapeutic ultrasonography. The Journal of Foot and Ankle Surgery. 2012; 51(6):706-710.

10. Ferrero G, Fabbro E, Orlandi D, Martini C, Lacelli F, Serafini $\mathrm{G}$ et al. Ultrasound-guided injection of plateletrich plasma in chronic Achilles and patellar tendinopathy. Journal of ultrasound. 2012; 15(4):260-266.

11. Charousset C, Zaoui A, Bellaiche L, Bouyer B. Are multiple platelet-rich plasma injections useful for treatment of chronic patellar tendinopathy in athletes? a prospective study. The American journal of sports medicine. 2014. 0363546513519964.

12. Sánchez M, Guadilla J, Fiz N, Andia I. Ultrasoundguided platelet-rich plasma injections for the treatment of osteoarthritis of the hip. Rheumatology. 2012; 51(1):144150. 